

\title{
AN ADAPTIVELY WEIGHTED GALERKIN FINITE ELEMENT METHOD FOR BOUNDARY VALUE PROBLEMS
}

\author{
Yifei Sun AND Chad R. WestPhaL
}

\begin{abstract}
We introduce an adaptively weighted Galerkin approach for elliptic problems where diffusion is dominated by strong convection or reaction terms. In such problems, standard Galerkin approximations can have unacceptable oscillatory behavior near boundaries unless the computational mesh is sufficiently fine. Here we show how adaptively weighting the equations within the variational problem can increase accuracy and stability of solutions on under-resolved meshes. Rather than relying on specialized finite elements or meshes, the idea here sets a flexible and robust framework where the metric of the variational formulation is adapted by an approximate solution. We give a general overview of the formulation and an algorithmic structure for choosing weight functions. Numerical examples are presented to illustrate the method.
\end{abstract}

\section{Introduction}

In this paper, we consider numerically approximating solutions to the diffusion, convection, reaction problem

$$
\left\{\begin{aligned}
-\varepsilon \Delta u+\boldsymbol{b} \cdot \nabla u+c u=f & \text { in } \Omega, \\
u=0 & \text { on } \partial \Omega .
\end{aligned}\right.
$$

Here, $u$ is the solution, $\Delta u$ and $\nabla u$ are the Laplacian and gradient of $u, \partial \Omega$ is the boundary of domain $\Omega$, and $f$ is a known data function. We assume that coefficients $c$ and $\varepsilon$ are positive constants, that $\boldsymbol{b}$ is a constant vector, and that boundary conditions are homogenous Dirichlet although nonzero boundary data or Neumann/mixed boundary conditions may easily be considered under appropriate smoothness assumptions. When $|\boldsymbol{b}| \gg \varepsilon$, we may consider this as a convectiondominated diffusion problem, which may have solutions with boundary layers downstream from $\boldsymbol{b}$. When $c \gg \varepsilon$ and the reaction term dominates, layer phenomena are also possible. It is well known that standard finite element and finite difference

Westphal's work was supported by the National Science Foundation under grant DMS-1216297. The authors wish to thank the anonymous reviewers for their helpful suggestions.

MSC2010: 65N30, 65N12, 35J20.

Keywords: finite element methods, convection-dominated diffusion, boundary layers, adaptive, weighted. 
approaches to such problems can yield solutions with undesirable overshoots and/or oscillatory behavior near these boundary layers when the computational mesh is not sufficiently resolved.

The difficulty associated with boundary layers is, of course, not limited to (1-1) but is evident in many applications where diffusive terms are dominated by convective or reactive terms. Throughout this paper, we assume sufficient regularity of the data and domain to ensure solutions are sufficiently smooth, which is a separate issue from boundary layer behavior.

There are many well studied numerical approaches to ameliorate layer effects. Through the use of specialized graded meshes $[24 ; 25 ; 11 ; 8]$ or adaptive mesh refinement [9], it is possible to develop a mesh that has sufficient resolution near the layers to resolve the solution and eliminate the effects of the high gradients on the solution in areas where the solution is smooth, which are commonly referred to as the "pollution effects". It is also possible to augment the weak form of the problem by adding mesh-dependent stabilization terms to the formulation $[16 ; 17 ; 2]$. These terms may or may not be consistent with the original problem, but they generally improve the solution on coarse meshes, and their influence diminishes as the mesh is resolved. The variational problem can also be modified through a Petrov-Galerkin formulation, where the test and trial spaces are different. This includes streamline upwind Petrov-Galerkin (SUPG) formulations $[5 ; 6 ; 18 ; 1]$ as well as methods with spaces enhanced by bubble functions [4]. Such problems have also been studied in the context of discontinuous Galerkin (DG) $[11 ; 15]$ and discontinuous Petrov-Galerkin (DPG) $[10 ; 13]$ methods. Here continuity requirements in the trial and test spaces are relaxed, and additional degrees of freedom on the element boundaries lead to additional jump conditions in the variational problem. Further comparisons on earlier work for such problems can be found in [22;12;14]. Broadly speaking, there are many ingredients in designing a finite element formulation (i.e., reformulating the equations, choosing/adapting the mesh, choosing test/trial spaces, etc.), and improvements on the standard Galerkin approach have been realized by many modifications and combinations of choices in the basic ingredients.

In this work, we introduce an adaptively weighted Galerkin finite element approach to (1-1) for cases exhibiting boundary layers. By generalizing the standard Galerkin weak form with weighted inner products, we may essentially redistribute the strength by which the variational problem is enforced across the domain. The use of weighted norms and weighted inner products is, of course, not a new idea. In [21], a weighted Galerkin formulation is used for a parabolic problem where the diffusion coefficient changes sign within the interior of the domain. A weighted Galerkin approach is coupled with a mapping technique in [23] to solve elliptic problems on unbounded domains. And in the least-squares finite element paradigm, using weighted norms to generalize $L^{2}$ residual minimization problems allows 
for robust treatment of problems with boundary singularities in weighted $H^{1}(\Omega)$ or $H$ (div) norms [19; 20; 7].

For problem (1-1), when the computational mesh is relatively coarse, weakening the problem near boundary layers in the right way can reduce or eliminate the pollution effects, stabilizing the numerical approximation. When the mesh is sufficiently fine to resolve the solution with no ill effects, the approach defaults to the standard Galerkin approach. Here, we explore this idea via an adaptive approach, whereby an approximate solution is used to generate a weight function to define a subsequent problem. While there are many successful methods in the literature, we are particularly motivated by practicality. In many cases, solutions tend to be smooth except for small regions representing a layer, and adopting an exotic approximation space to represent the global solution seems excessive. Generally, if it were computable, the interpolant of even simple finite element spaces would provide a sufficient approximation. Our approach is designed to generalize the standard Galerkin approach, where the mesh and trial space can be chosen based on resolving the features of the solution, and an initial approximation helps redefine the metric of an improved variational formulation. For simplicity, we describe our approach separately from adaptive mesh refinement though mesh adaptation can be used alongside our weighting approach. The weighted Galerkin approach here can also be viewed as a Petrov-Galerkin formulation, where the basis functions of the test space are generated adaptively, based on an approximate solution.

The organization of this paper is as follows. In the following section, we introduce the idea of an adaptively weighted variational problem and describe the construction of an appropriate weight function. Numerical results are given in Section 3, and a brief look at the how the coercivity of the weighted bilinear form is enhanced from the standard approach is given in Section 4.

\section{Weighted Galerkin formulation}

Throughout this paper, we use standard notation for the $L^{2}(\Omega)^{d}$ norm, $\|\cdot\|$, and inner product, $(\cdot, \cdot)$, and use $\|\cdot\|_{H^{k}}$ to denote the norm corresponding to the Sobolev space $H^{k}(\Omega)^{d}$. The space of continuous functions on $\Omega$ is denoted by $C^{0}(\Omega)^{d}$, and we recall that for $\phi \in H^{1}(\Omega)^{d}$ and $\psi \in C^{0}(\Omega)^{d}$ we have $\phi \psi \in H^{1}(\Omega)^{d}$ for $d=1,2,3$. When the dimension of the problem is understood in context, we drop the $d$ superscript. Since the relative balance between diffusion, convection, and reaction terms in (1-1) determines the behavior of the solution, for the remainder of this paper, we take $\varepsilon=1$ without loss of generality.

Defining the space $V=\left\{v \in H^{1}(\Omega): v=0\right.$ on $\left.\partial \Omega\right\}$ and the bilinear form

$$
a(u, v):=(\nabla u, \nabla v)+(\boldsymbol{b} \cdot \nabla u, v)+(c u, v),
$$


the standard variational formulation of (1-1) for a given $f \in L^{2}(\Omega)$ is to find $u \in V$ such that $a(u, v)=(f, v)$ for all $v \in V$. The discrete Galerkin formulation is analogous: define a finite element space, $V^{h} \subset V$, and find $u^{h} \in V^{h}$ such that

$$
a\left(u^{h}, v^{h}\right)=\left(f, v^{h}\right) \quad \text { for all } v^{h} \in V^{h} .
$$

Since the main idea in this paper is in modifying the variational framework to achieve better global approximations on relatively coarse resolutions, the choice of the finite element space can be made according to its approximation properties in the interior of the domain or according to simplicity or availability of code. In Section 3, we give numerical results using simple conforming piecewise polynomial spaces. In many cases, solutions tend to be relatively smooth up to boundary layers, and finding accurate approximations up to boundary layers is desirable.

Now let $w \in C^{0}(\Omega)$ be a weight function such that $0<w_{\min } \leq w(\boldsymbol{x}) \leq 1$. We define a weighted bilinear form by multiplying each side of the PDE in (1-1) by $w v$ and integrating by parts:

$$
\begin{aligned}
(f, w v) & =(-\nabla \cdot \nabla u, w v)+(\boldsymbol{b} \cdot \nabla u, w v)+(c u, w v) \\
& =(\nabla u, \nabla(w v))+(\boldsymbol{b} \cdot \nabla u, w v)+(c u, w v) \\
& =(\nabla u, w \nabla v+v \nabla w)+(\nabla u, w v \boldsymbol{b})+(c u, w v) \\
& =(\nabla u, w \nabla v)+(\nabla u,(\nabla w+w \boldsymbol{b}) v)+(c u, w v)=: W(u, v) .
\end{aligned}
$$

The discrete weighted variational formulation of (1-1) is thus to find $u^{h} \in V^{h}$ such that

$$
W\left(u^{h}, v^{h}\right)=\left(w f, v^{h}\right) \quad \text { for all } v^{h} \in V^{h} .
$$

At this point, the weight function need only be sufficiently smooth and positive. Within these requirements, the construction of the weight function is motivated by producing a more robust numerical approximation. Notice that

$$
W(v, v)=\left\|w^{1 / 2} \nabla v\right\|^{2}+(\nabla v,(\nabla w+w \boldsymbol{b}) v)+c\left\|w^{1 / 2} v\right\|^{2},
$$

which indicates in general that choosing $(\nabla w+w \boldsymbol{b})$ small will make the cross term small and $W(v, v)$ will more resemble a weighted $H^{1}(\Omega)$ measure. In Section 4, we explore the connection between $w$ and the coercivity of $W(\cdot, \cdot)$. In a practical sense, we may view the role of $w$ in (2-3) as being able to dampen the effect of large values of $\left|\nabla u^{h}\right|$. Formally, we know that asymptotic solutions of (1-1) near boundaries may have terms of the form

$$
e^{-k x} \text { and } e^{ \pm \sqrt{c} x}
$$

where $k=|\boldsymbol{b}|$, and thus, boundary layer solutions may change on the order of $\max \left\{e^{k h}, e^{\sqrt{c} h}\right\}$ across individual elements of mesh size $h$. This dramatic growth 
of the solution is often problematic, and we are motivated to choose $w$ small in these regions to decouple the effects from the rest of the problem. In other words, in regions where $\left|\nabla u^{h}\right|$ is relatively large, $w$ should be chosen to make $(\nabla w+w \boldsymbol{b})$ small. When the mesh size is small enough to reasonably represent the solution, the standard Galerkin approach yields acceptable results. We now describe an adaptive approach to generate an appropriate weight function, based on the coefficients from the original PDE, an initial approximation to the solution, and the mesh size.

If $u_{\text {old }}^{h}$ represents an initial approximation to the solution to (1-1), we simply want to choose $w$ large/small where the magnitude of $\nabla u_{\text {old }}^{h}$ is small/large. While there are many empirical approaches to constructing $w$ from this guiding principle, we describe one here that is simple to implement and tends to give robust results. Let $\Omega^{h}$ be a triangulation of the domain with elements denoted by $\tau_{i}$, for $i$ ranging from 1 to the number of elements, and choose the approximation space $V^{h}$. Our approach is given by the following algorithm:

(1) Start: Initially set $w=1$ uniformly.

(2) Solve: Obtain an initial solution $u_{\text {old }}^{h}$ by solving (2-2).

(3) Construct weight:

- For each element, $\tau_{i}$, compute $d_{i}:=\left\|\nabla u_{\text {old }}^{h}\right\|_{\tau_{i}}$.

- Denote the minimum/maximum values of this set by $d_{\min }=\min _{\tau_{i} \in \Omega^{h}} d_{i}$ and $d_{\max }=\max _{\tau_{i} \in \Omega^{h}} d_{i}$.

- Set $\widehat{w}_{i}=1-\left(1-w_{\min }\right)\left(d_{i}-d_{\min }\right) /\left(d_{\max }-d_{\min }\right)$.

- Construct $w(\boldsymbol{x}) \in C^{0}(\Omega)$ as a piecewise linear function from elementwise values $\widehat{w}_{i}$. See Section 3 for details.

(4) Re-solve: Using $w$, find $u^{h}$ by solving problem (2-3).

Algorithm 1. Adaptively weighted Galerkin approximation.

This basic approach can be modified to accommodate other features. As described, this requires two PDE solves on the same mesh. However, it is straightforward to refine the mesh, either locally or globally, between steps (2) and (4) and use the weight from the coarse solve to enhance the fine solve. Similarly, nonlinear or time-dependent problems that rely on an iterative approach based on an approximate solution can incorporate steps (3) and (4) to the iterative method.

In the next section, we show numerical test problems and give details on constructing $w \in C^{0}(\Omega)$ from the elementwise values $\widehat{w}_{i}$.

\section{Numerical results}

In this section, we consider two test problems in 1D where the features of the weighted Galerkin approach can clearly be seen. We then provide an example in $2 \mathrm{D}$ that shows how the approach can be incorporated into a more realistic setting. 

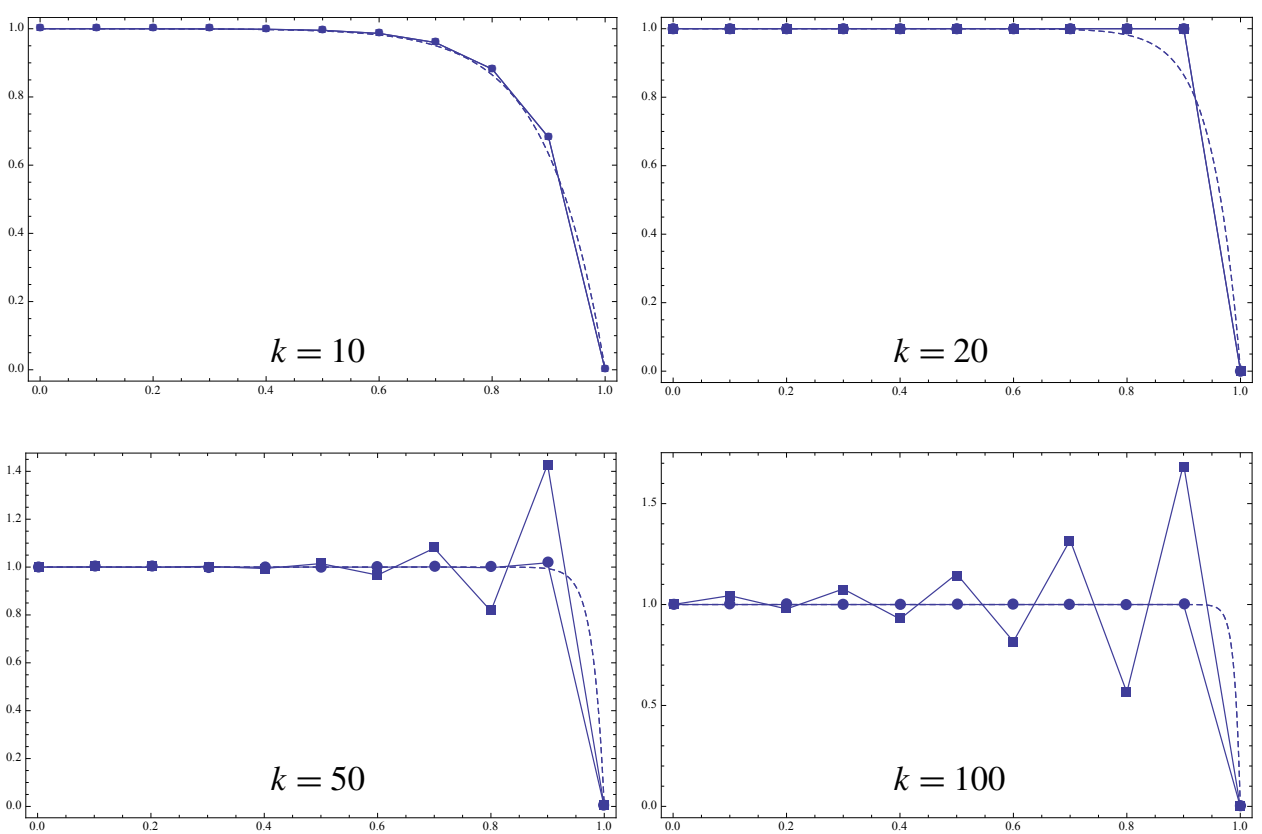

Figure 1. Standard Galerkin (squares) and weighted Galerkin (circles) approximations to (3-1) compared with exact solutions (dashed), for $n=10$ and $k=10,20,50,100$.

3.1. 1D convection-dominated. For this first example, we consider the interplay between diffusion and convection in the ODE model problem

$$
\left\{\begin{aligned}
-u^{\prime \prime}+k u^{\prime} & =0 \quad \text { in }(0,1), \\
u(0) & =1, \\
u(1) & =0
\end{aligned}\right.
$$

which has exact solution $u(x)=\left(e^{k x}-e^{k}\right) /\left(1-e^{k}\right)$.

We discretize $\Omega=(0,1)$ into $n$ evenly spaced subintervals of mesh size $h=1 / n$, with nodes $0=x_{0}, x_{1}, \ldots, x_{n}=1$, and define $V^{h}$ as the set of continuous piecewise linear functions satisfying the boundary conditions in (3-1). We follow the basic approach in Algorithm 1, using $w_{\min }=e^{-h k}$, and we construct $w(x)$ as a piecewise linear function on the existing mesh by setting nodal values according to

$$
w\left(x_{i}\right)= \begin{cases}\widehat{w}_{1} & \text { for } i=0, \\ \min \left\{\widehat{w}_{i}, \widehat{w}_{i+1}\right\} & \text { for } i=1,2, \ldots, n-1, \\ \widehat{w}_{n} & \text { for } i=n .\end{cases}
$$

For $k \leq 2 n$, the standard Galerkin approach does not give overshoots or oscillatory behavior, but as $k$ increases beyond this, such undesirable behavior occurs. This range also corresponds to the spectrum of the system matrix, which is all real 

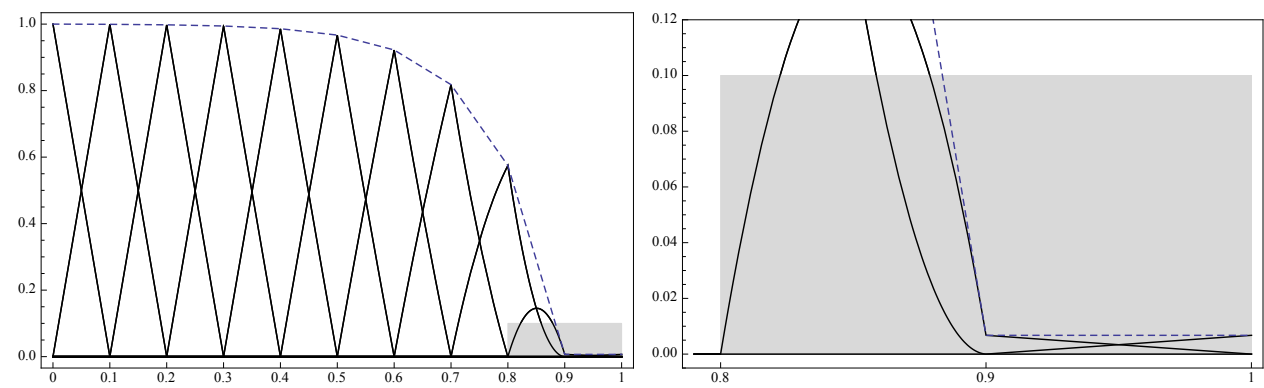

Figure 2. Basis functions $\psi_{i}=w \phi_{i}$ : on the entire domain (left) and detail near the boundary layer (right).

for $k \leq 2 n$ and complex for $k>2 n$. We are thus free to fix the value of $n$ and vary $k$ to explore the possible numerical behavior of the boundary layer near $x=1$. In Figure 1, results are shown for $n=10$ and $k=10,20,50,100$. Improved results can clearly be seen in all cases, where for small $k$ the weighted approach is essentially the same as the standard approach, and as $k$ increases, the weighted approach appropriately isolates the behavior of the solution near the boundary layer.

One way to view the role of the weight functions is as a Petrov-Galerkin formulation, where the basis functions on the test space are created by weighting the basis of the trial space. For example, here $\left\{\phi_{i}\right\}_{i=0}^{n}$ represents the standard piecewise linear basis and $u^{h} \in V^{h}=\operatorname{span}\left\{\phi_{i}\right\}$. Given a weight function, $w$, the weighted Galerkin method is to find $u^{h} \in V^{h}$ such that

$$
a\left(u^{h}, z^{h}\right)=\left(f, z^{h}\right) \quad \text { for all } z^{h} \in W^{h},
$$

where $v^{h} \in W^{h}=\operatorname{span}\left\{\psi_{i}\right\}$, where $\psi_{i}=w \phi_{i}$ for each $i=0,1, \ldots, n$. Figure 2 shows this basis for the $1 \mathrm{D}$ convection-dominated problem with $n=10$ and $k=50$. Near the boundary layer, there is a clear upwinding effect, and in places where the solution is smooth, the basis functions for $W^{h}$ and $V^{h}$ are essentially the same. Our adaptive approach tends to resemble the SUPG approach in areas where $\left|\nabla u^{h}\right|$ is large and the standard Galerkin approach otherwise.

\subsection{D reaction-dominated. Consider the reaction-dominated diffusion ODE}

$$
\left\{\begin{aligned}
-u^{\prime \prime}+c u & =c \quad \text { in }(0,1), \\
u(0) & =0, \\
u(1) & =0,
\end{aligned}\right.
$$

which yields a solution, $u(x)=1-\left(e^{\sqrt{c}(1-x)}+e^{\sqrt{c} x}\right) /\left(1+e^{\sqrt{c}}\right)$, that develops boundary layers at $x=0$ and $x=1$ for $c \gg 1$. It's easy to see that as $c \rightarrow \infty$ the solution approaches $u=1$ for $x \in(0,1)$, yet the boundary conditions require $u(0)=u(1)=0$. We use the weighted Galerkin approach as described above but 

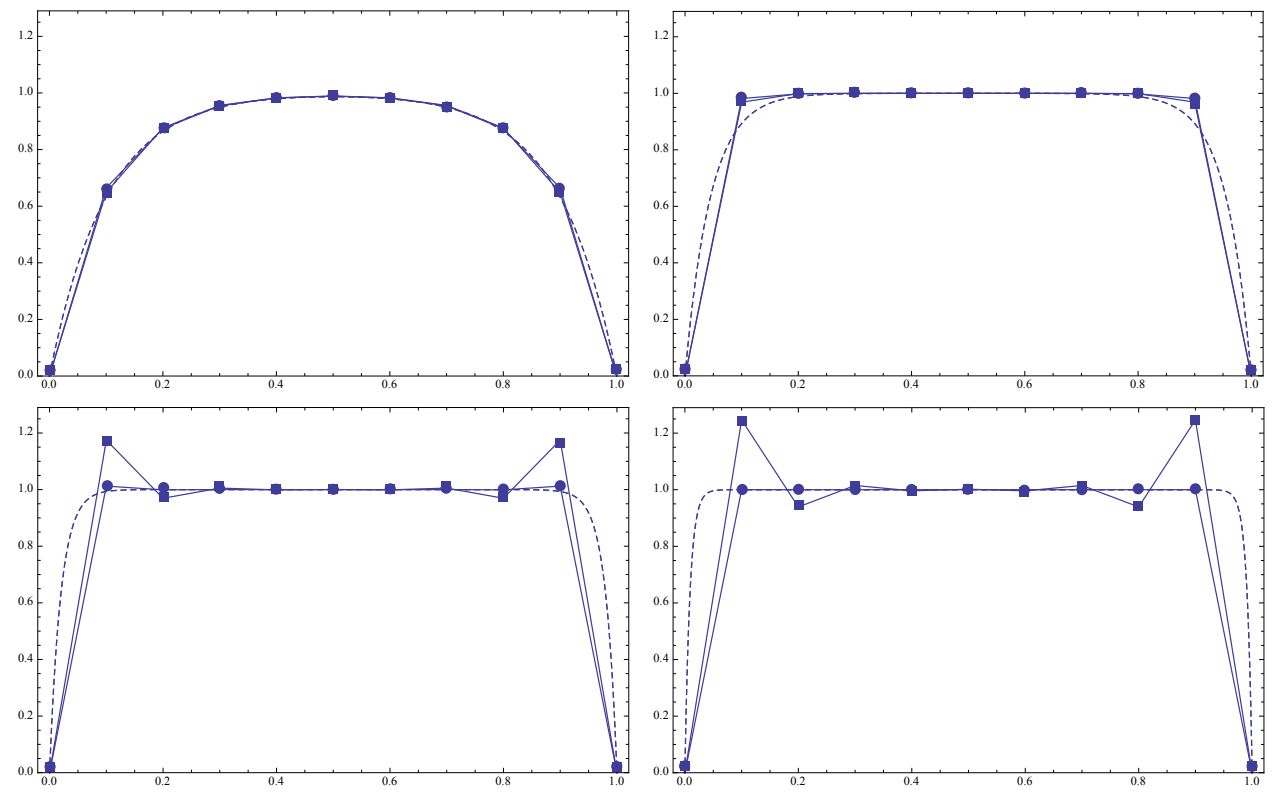

Figure 3. Standard Galerkin (squares) and weighted Galerkin (circles) approximations to (3-2) for $c=100,500,2500,12500$ with exact solutions (dashed).

with $w_{\min }=e^{-\sqrt{c} / n}$, a choice motivated by the change of the asymptotic solution in the elements nearest each boundary.

Figure 3 shows results qualitatively similar to those in Figure 1. As $c$ increases, boundary layers form near $x=0$ and $x=1$. For $n$ small relative to $\sqrt{c}$, both the standard and weighted Galerkin approaches give accurate approximations, but for large values of $\sqrt{c}$, the weighted approach yields better solutions.

3.3. $2 D$ convection-dominated. As an example in $2 \mathrm{D}$, we consider

$$
-\Delta u+\boldsymbol{b} \cdot \nabla u=0 \quad \text { in } \Omega \quad \text { with } \boldsymbol{b}=\frac{200}{\sqrt{x^{2}+y^{2}}}\left[\begin{array}{r}
-y \\
x
\end{array}\right] .
$$

We use $\Omega=(0,1)^{2}$ with zero Dirichlet boundary conditions on the north, east, and west boundaries and $u(x, 0)=16 x^{2}(1-x)^{2}$ on the south boundary. Here, $\boldsymbol{b}$ represents a convection term of magnitude $k=|\boldsymbol{b}|=200$, which is in a counterclockwise circular rotation. The solution forms a boundary layer on the west boundary (see Figure 4).

We discretize $\Omega$ using a uniform mesh of triangles of size $h$ and use standard $P_{1}$ elements for the approximation $u^{h}$. As in the previous examples, we follow the structure of Algorithm 1. To construct $w(\boldsymbol{x})$ as a $P_{1}$ finite element function on the existing mesh, we choose $w_{\min }=e^{-k h}$ and the nodal values of $w$ as the minimum of $d_{i}$ on all adjacent triangles. We then find $u^{h}$ as the solution of (2-3). Figure 4 


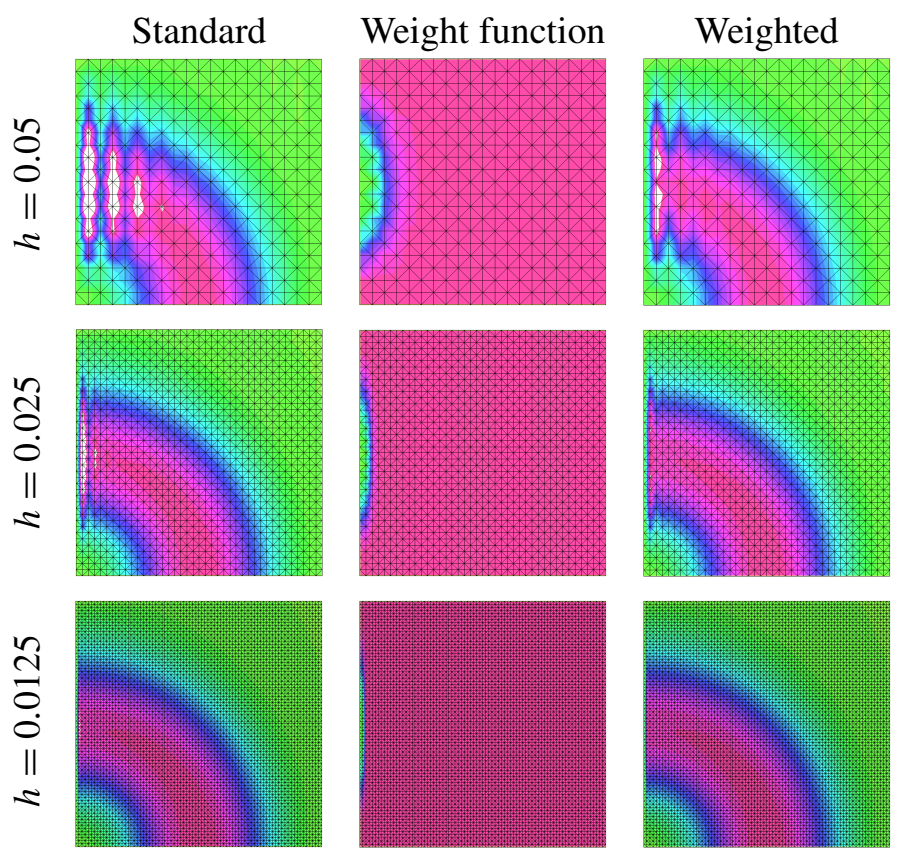

Figure 4. Numerical approximations and adaptively generated weight functions for 2D convection-dominated diffusion problem. Values in $[0,1]$ are shown in color (green corresponds to 0 and pink corresponds to 1) while values greater than 1 are in white. Overshoot values are given in Table 1 .

shows plots of the approximations, comparing the standard Galerkin solution and the weighted Galerkin solution for three mesh resolutions. Note that the boundary conditions dictate that $u \leq 1$, and we take values exceeding $u=1$ to be considered an overshoot. Weighted Galerkin solutions show significantly less oscillatory behavior than the standard approach. Table 1 shows overshoot values for both approaches.

In Figure 5, we compare the weighted and standard Galerkin approaches by plotting the $L^{2}$ error, $\left\|u^{h}-u^{*}\right\|$, and the $H^{1}$ seminorm error, $\left\|\nabla\left(u^{h}-u^{*}\right)\right\|$, for increasing mesh resolution $(n=10,20,40,80)$. We use the numerical solution on a very fine mesh, $u^{*}(n=600)$, as a proxy for the exact solution. Both methods approach the asymptotic optimal rates of $O\left(h^{2}\right)$ and $O(h)$, but the weighted approach gives better approximations on under-resolved meshes.

\begin{tabular}{lcc}
$h$ & Standard scheme & Weighted scheme \\
\hline 0.05 & 1.70 & 1.15 \\
0.025 & 1.44 & 1.04 \\
0.0125 & 1.10 & 1.00
\end{tabular}

Table 1. Overshoot values for numerical approximations, $\max _{\boldsymbol{x} \in \Omega}\left|u^{h}\right|$. 

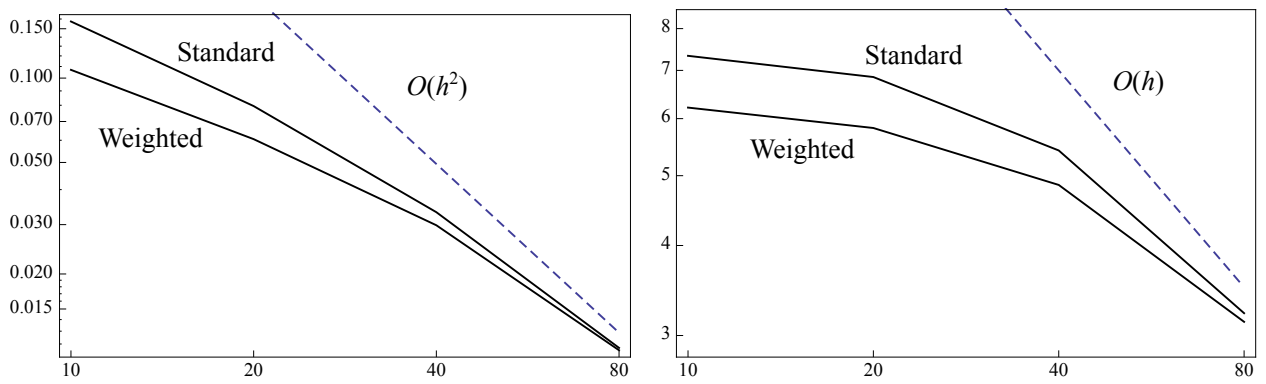

Figure 5. $L^{2}$ norm and $H^{1}$ seminorm errors for $2 \mathrm{D}$ convection-dominated diffusion problem.

\section{On coercivity}

We briefly explore the connection between the behavior of convection-dominated problems and the coercivity of the variational problem. Understanding when coercivity is lost and the effects on the linear systems leads to a better understanding of how to construct an improved variational problem using weighted inner products.

Definition 4.1. A bilinear form $a(\cdot, \cdot)$ on a normed linear space $H$ is said to be coercive on $V \subseteq H$ if there exists $\alpha>0$ such that

$$
a(v, v) \geq \alpha\|v\|_{H}^{2} \quad \text { for all } v \in V .
$$

Coercivity is of great interest since, if $(H,(\cdot, \cdot))$ is a Hilbert space, $V$ is a subspace of $H$, and $a(\cdot, \cdot)$ is an inner product on $V$, then $(V, a(\cdot, \cdot))$ need not be complete if $a(\cdot, \cdot)$ is not coercive [3]. When many standard approaches are employed for (1-1), solutions exhibit the well known numerical instability of oscillatory behavior near boundary layers for $h$ not sufficiently fine (e.g., see [17; $12 ; 22 ; 14])$.

The Galerkin variational formulation of (1-1) for a given $f \in L^{2}(\Omega)$ is to find a $u \in V$ such that

$$
a(u, v)=(f, v) \text { for all } v \in V,
$$

where $a(u, v):=(\nabla u, \nabla v)+(\boldsymbol{b} \cdot \nabla u, v)+(c u, v)$.

In the following, we assume constant $\boldsymbol{b}$ and examine coercivity of $a(\cdot, \cdot)$ in the absence of boundary conditions:

Proposition 4.2. If we choose $H=V=H^{1}(\Omega)$, then coercivity holds for $a(\cdot, \cdot)$ if and only if $k<2 \sqrt{c}$, where $k=|\boldsymbol{b}|$.

Proof. We first prove the "if" part. Since $k<2 \sqrt{c}$, we can find $0<c_{0}<\min \{1, c\}$ such that

$$
k \leq 2 \sqrt{\left(1-c_{0}\right)\left(c-c_{0}\right)} .
$$


By the Cauchy-Schwarz inequality, we have that

$$
\begin{aligned}
|(\boldsymbol{b} \cdot \nabla v, v)| & \leq\|\boldsymbol{b} \cdot \nabla v\|\|v\| \leq k \cdot\|\nabla v\|\|v\| \\
& \leq 2 \sqrt{\left(1-c_{0}\right)\left(c-c_{0}\right)} \cdot\|\nabla v\|\|v\| \\
& \leq\left(1-c_{0}\right)\|\nabla v\|^{2}+\left(c-c_{0}\right)\|v\|^{2} .
\end{aligned}
$$

Thus,

$$
\left(1-c_{0}\right)\|\nabla v\|^{2}+(\boldsymbol{b} \cdot \nabla v, v)+\left(c-c_{0}\right)\|v\|^{2} \geq 0
$$

which gives us

$$
\|\nabla v\|^{2}+(\boldsymbol{b} \cdot \nabla v, v)+c\|v\|^{2} \geq c_{0}\left(\|\nabla v\|^{2}+\|v\|^{2}\right)=c_{0}\|v\|_{H^{1}}^{2} .
$$

To prove the "only if" part, we need to show that, for every $c_{0}>0$, we can always find $v \in H^{2}(\Omega) \cap V$ such that

$$
a(v, v)<c_{0}\|v\|_{H^{1}}^{2} .
$$

Since $k \geq 2 \sqrt{c}$, for all $c_{0}>0$ satisfying $c_{0}<\min \{1, c\}$, we have

$$
2 \sqrt{\left(c-c_{0}\right)\left(1-c_{0}\right)}<k .
$$

We let $\boldsymbol{x} \in \mathbb{R}^{d}$, where $d$ is the dimension of $\boldsymbol{b}$, and

$$
\boldsymbol{a}=-\frac{1}{k} \sqrt{\frac{c-c_{0}}{1-c_{0}}} \boldsymbol{b}
$$

Then if we choose $v=e^{a \cdot x}$, we will get

$$
\nabla v=v \boldsymbol{a}
$$

and

$$
\boldsymbol{b} \cdot \nabla v=(\boldsymbol{a} \cdot \boldsymbol{b}) v=k^{2} \cdot\left(-\frac{1}{k} \sqrt{\frac{c-c_{0}}{1-c_{0}}} v\right)=-k \sqrt{\frac{c-c_{0}}{1-c_{0}}} v
$$

We notice (4-2) and (4-3) give us the following relation:

$$
|\nabla v|=\sqrt{\frac{c-c_{0}}{1-c_{0}}}|v|
$$

Thus,

$$
\begin{aligned}
\|\nabla v\|=\left(\int_{\Omega}(\nabla v \cdot \nabla v)\right)^{1 / 2}=\left(\int_{\Omega}|\nabla v|^{2}\right)^{1 / 2} & \\
= & \sqrt{\frac{c-c_{0}}{1-c_{0}}}\left(\int_{\Omega} v^{2}\right)^{1 / 2}=\sqrt{\frac{c-c_{0}}{1-c_{0}}}\|v\|,
\end{aligned}
$$


and

$$
\begin{aligned}
(\boldsymbol{b} \cdot \nabla v, v) & =-k \sqrt{\frac{c-c_{0}}{1-c_{0}}}\|v\|^{2} \\
& <-2 \sqrt{\left(c-c_{0}\right)\left(1-c_{0}\right)} \sqrt{\frac{c-c_{0}}{1-c_{0}}}\|v\|^{2} \\
& =-2\left(c-c_{0}\right)\|v\|^{2} .
\end{aligned}
$$

By plugging (4-4) into (4-5), we have

$$
(\boldsymbol{b} \cdot \nabla v, v)<-2\left(1-c_{0}\right)\|\nabla v\|^{2} .
$$

After adding up (4-5) and (4-6), we get

$$
(\boldsymbol{b} \cdot \nabla v, v)<-\left(c-c_{0}\right)\|v\|^{2}-\left(1-c_{0}\right)\|\nabla v\|^{2},
$$

which leads to

$$
a(v, v)=\|\nabla v\|^{2}+(\boldsymbol{b} \cdot \nabla v, v)+c\|v\|^{2}<c_{0}\left(\|\nabla v\|^{2}+\|v\|^{2}\right)=c_{0}\|v\|_{H^{1}}^{2} .
$$

This shows, in part, why convection-dominated problems using the standard Galerkin approach may perform poorly in practice. To address how the weighted variational approach improves the outlook, we recall (2-4),

$$
W(v, v)=\left\|w^{1 / 2} \nabla v\right\|^{2}+(\nabla v,(\nabla w+w \boldsymbol{b}) v)+c\left\|w^{1 / 2} v\right\|^{2} .
$$

When $|\boldsymbol{b}|$ is large and $w=1$, it is clear that the cross term can dominate the expression. To illustrate the impact of this term, let $k=|\boldsymbol{b}|$ and assume that $w$ is such that

$$
|\nabla w+w \boldsymbol{b}| \leq \theta|w \boldsymbol{b}|=\theta k|w|
$$

for the smallest $\theta \geq 0$ possible. The unweighted case $(w=1)$ corresponds to $\theta=1$, and we can expect $\theta \rightarrow 0$ as $\nabla w+w \boldsymbol{b} \rightarrow \mathbf{0}$ whenever $w$ is uniformly bounded away from zero (i.e., $w \geq w_{\min }>0$ ). The construction of $w$ described in this paper leads to $\nabla w \cdot \boldsymbol{b}<0$ (i.e., boundary layers form downstream of $\boldsymbol{b}$, where $w$ increases in the opposite direction to $\boldsymbol{b})$, and thus, typically $\theta \in[0,1]$. Experimental evidence with the construction of $w$ in the test problem in Section 3.1 shows that in regions of $\Omega$ near boundary layers elementwise values of $\theta$ are in $[0,1)$ while throughout the interior of $\Omega$ values of $\theta$ are near 1 . 
Recalling the general inequality $x y \leq(\epsilon / 2) x^{2}+(1 / 2 \epsilon) y^{2}$ for any $\epsilon>0$ and the Cauchy-Schwarz inequality, the coercivity of $W(\cdot, \cdot)$ then follows from

$$
\begin{aligned}
W(v, v) & =\left\|w^{1 / 2} \nabla v\right\|^{2}+(\nabla v,(\nabla w+w \boldsymbol{b}) v)+c\left\|w^{1 / 2} v\right\|^{2} \\
& \geq\left\|w^{1 / 2} \nabla v\right\|^{2}-\theta k\left\|w^{1 / 2} \nabla v\right\|\left\|w^{1 / 2} v\right\|+c\left\|w^{1 / 2} v\right\|^{2} \\
& \geq\left\|w^{1 / 2} \nabla v\right\|^{2}-\theta k\left(\frac{\epsilon}{2}\left\|w^{1 / 2} \nabla v\right\|^{2}+\frac{1}{2 \epsilon}\left\|w^{1 / 2} v\right\|^{2}\right)+c\left\|w^{1 / 2} v\right\|^{2} \\
& =\left(1-\frac{\theta k \epsilon}{2}\right)\left\|w^{1 / 2} \nabla v\right\|^{2}+\left(c-\frac{\theta k}{2 \epsilon}\right)\left\|w^{1 / 2} v\right\|^{2} \\
& \geq c_{0}\left(\left\|w^{1 / 2} \nabla v\right\|^{2}+\left\|w^{1 / 2} v\right\|^{2}\right),
\end{aligned}
$$

where $0<c_{0}=\min \{1-\theta k \epsilon / 2, c-\theta k / 2 \epsilon\}$ when $\epsilon \in[\theta k / 2 c, 2 / \theta k]$ and $k \leq 2 \sqrt{c} / \theta$. This somewhat formal view shows that the weighted variational problem induces a weighted $H^{1}$ norm that may be more desirable than the standard $H^{1}$ measure since when $\theta<1$ the coercivity of $W(\cdot, \cdot)$ will hold for a larger range of $k$ than the standard Galerkin approach, which requires $k \leq 2 \sqrt{c}$.

When coercivity holds, both the standard Galerkin and weighted Galerkin approaches can easily be shown to have optimal-order error bounds. That is, when (1-1) has full regularity, we have

$$
\left\|u-u^{h}\right\|=C h^{2}\|u\|_{H^{2}} \quad \text { and } \quad\left\|\nabla u-\nabla u^{h}\right\|=C h\|u\|_{H^{2}},
$$

where $u$ is the exact solution, $u^{h}$ is the numerical approximation with mesh size $h$ (see, e.g., Figure 5). When the coercivity bound holds for a wider range of parameters, it is reasonable to expect more robust numerical results.

\section{Conclusion}

The weighted scheme we present in this paper seeks to provide a natural reformulation of the variational approach that has an underlying metric adapted to the specific problem. The approach tends to induce an upwinding effect and is flexible in that it does not require specialized meshing or the use of exotic elements. It is possible also to extend the idea to problems with boundary singularities, where overall convergence rates are affected by the loss of smoothness. This study is the subject of a forthcoming investigation.

\section{References}

[1] P. B. Bochev, M. D. Gunzburger, and J. N. Shadid, Stability of the SUPG finite element method for transient advection-diffusion problems, Comput. Methods Appl. Mech. Engrg. 193 (2004), no. 23-26, 2301-2323. MR 2005a:65100 Zbl 1067.76563 
[2] P. B. Bochev and K. Peterson, A parameter-free stabilized finite element method for scalar advection-diffusion problems, Cent. Eur. J. Math. 11 (2013), no. 8, 1458-1477. MR 3056328 Zbl 1273.65173

[3] S. C. Brenner and L. R. Scott, The mathematical theory of finite element methods, 3rd ed., Texts in Applied Mathematics, no. 15, Springer, New York, 2008. MR 2008m:65001 Zbl 1135.65042

[4] F. Brezzi and A. Russo, Choosing bubbles for advection-diffusion problems, Math. Models Methods Appl. Sci. 4 (1994), no. 4, 571-587. MR 95h:76079 Zbl 0819.65128

[5] A. N. Brooks and T. J. R. Hughes, Streamline upwind/Petrov-Galerkin methods for advection dominated flows, Proceedings of the Third International Conference on Finite Elements in Flow Problems (Banff, AB, 1980), vol. 2, University of Calgary, Calgary, AB, 1980, edited by D. H. Norrie, pp. 283-292. Zbl 0449.76077

[6] A. N. Brooks and T. J. R. Hughes, Streamline upwind/Petrov-Galerkin formulations for convection dominated flows with particular emphasis on the incompressible Navier-Stokes equations, Comput. Methods Appl. Mech. Engrg. 32 (1982), no. 1-3, 199-259. MR 83k:76005 Zbl 0497.76041

[7] Z. Cai and C. R. Westphal, A weighted H(div) least-squares method for second-order elliptic problems, SIAM J. Numer. Anal. 46 (2008), no. 3, 1640-1651. MR 2008m:65314 Zbl 1168.65069

[8] G. F. Carey and H. T. Dinh, Grading functions and mesh redistribution, SIAM J. Numer. Anal. 22 (1985), no. 5, 1028-1040. MR 86h:65123 Zbl 0577.65076

[9] W. Castaings and I. M. Navon, Mesh refinement strategies for solving singularly perturbed reaction-diffusion problems, Comput. Math. Appl. 41 (2001), no. 1-2, 157-176. MR 1808513 Zbl 0984.65125

[10] P. Causin, R. Sacco, and C. L. Bottasso, Flux-upwind stabilization of the discontinuous PetrovGalerkin formulation with Lagrange multipliers for advection-diffusion problems, Math. Model. Numer. Anal. 39 (2005), no. 6, 1087-1114. MR 2006j:76085 Zbl 1084.65105

[11] B. Cockburn, B. Dong, and J. Guzmán, Optimal convergence of the original DG method for the transport-reaction equation on special meshes, SIAM J. Numer. Anal. 46 (2008), no. 3, 1250-1265. MR 2009c:65299 Zbl 1168.65058

[12] R. Codina, Comparison of some finite element methods for solving the diffusion-convectionreaction equation, Comput. Methods Appl. Mech. Engrg. 156 (1998), no. 1-4, 185-210. MR 99d:65280 Zbl 0959.76040

[13] L. Demkowicz and J. Gopalakrishnan, A class of discontinuous Petrov-Galerkin methods, II: Optimal test functions, Numer. Methods Partial Differential Equations 27 (2011), no. 1, 70-105. MR 2011k:65155 Zbl 1208.65164

[14] J. Douglas, Jr. and T. F. Russell, Numerical methods for convection-dominated diffusion problems based on combining the method of characteristics with finite element or finite difference procedures, SIAM J. Numer. Anal. 19 (1982), no. 5, 871-885. MR 84b:65093 Zbl 0492.65051

[15] H. Egger and J. Schöberl, A hybrid mixed discontinuous Galerkin finite-element method for convection-diffusion problems, IMA J. Numer. Anal. 30 (2010), no. 4, 1206-1234. MR 2011j: 65271 Zbl 1204.65133

[16] L. P. Franca, S. L. Frey, and T. J. R. Hughes, Stabilized finite element methods, I: Application to the advective-diffusive model, Comput. Methods Appl. Mech. Engrg. 95 (1992), no. 2, 253-276. MR 92m:76089 Zbl 0759.76040

[17] L. P. Franca, T. E. Tezduyar, and A. Masud (eds.), Finite element methods: 1970's and beyond, Centro Internacional de Métodos Numéricos en Ingeniería, Barcelona, 2004. 
[18] T. J. R. Hughes and A. N. Brooks, A theoretical framework for Petrov-Galerkin methods with discontinuous weighting functions: application to the streamline-upwind procedure, Finite elements in fluids (Banff, AB, 1980), vol. 4, Wiley, New York, 1982, edited by R. H. Gallager et al., pp. 47-65. MR 84d:76001 Zbl 0571.76002

[19] E. Lee, T. A. Manteuffel, and C. R. Westphal, Weighted-norm first-order system least squares (FOSLS) for problems with corner singularities, SIAM J. Numer. Anal. 44 (2006), no. 5, 1974 1996. MR 2008a:65221 Zbl 1129.65087

[20] _ Weighted-norm first-order system least-squares (FOSLS) for div/curl systems with three dimensional edge singularities, SIAM J. Numer. Anal. 46 (2008), no. 3, 1619-1639. MR 2009c:65316 Zbl 1170.65095

[21] H. Lu, Galerkin and weighted Galerkin methods for a forward-backward heat equation, Numer. Math. 75 (1997), no. 3, 339-356. MR 97m:65180 Zbl 0876.65071

[22] K. W. Morton, Numerical solution of convection-diffusion problems, Applied Mathematics and Mathematical Computation, no. 12, Chapman \& Hall, London, 1996. MR 98b:65004 Zbl 0861.65070

[23] H.-S. Oh, B. Jang, and Y. Jou, The weighted Ritz-Galerkin method for elliptic boundary value problems on unbounded domains, Numer. Methods Partial Differential Equations 19 (2003), no. 3, 301-326. MR 2004c:65148 Zbl 1021.65058

[24] G. I. Shishkin, Grid approximation of singularly perturbed boundary value problems with a regular boundary layer, Soviet J. Numer. Anal. Math. Modelling 4 (1989), no. 5, 397-418. MR 91b:65138 Zbl 0825.65057

[25] _ Grid approximation of singularly perturbed boundary value problems with convective terms, Soviet J. Numer. Anal. Math. Modelling 5 (1990), no. 2, 173-187. MR 92h:65142 Zbl 0816.65051

Received October 27, 2013. Revised June 12, 2014.

YIFEI SUN: yifei@cims.nyu.edu

Courant Institute of Mathematical Sciences, New York University, New York, NY 10012,

United States

CHAD R. WestPhal: westphac@wabash.edu

Department of Mathematics and Computer Science, Wabash College, Crawfordsville, IN 47933, United States 


\title{
Communications in Applied Mathematics and Computational Science
}

\author{
msp.org/camcos
}

EDITORS

MANAGING EDITOR

John B. Bell

Lawrence Berkeley National Laboratory, USA

jbbell@lbl.gov

\section{BOARD OF EDITORS}

\begin{tabular}{|c|c|c|c|}
\hline Marsha Berger & $\begin{array}{l}\text { New York University } \\
\text { berger@cs.nyu.edu }\end{array}$ & Ahmed Ghoniem & $\begin{array}{l}\text { Massachusetts Inst. of Technology, USA } \\
\text { ghoniem@mit.edu }\end{array}$ \\
\hline Alexandre Chorin & $\begin{array}{l}\text { University of California, Berkeley, USA } \\
\text { chorin@math.berkeley.edu }\end{array}$ & Raz Kupferman & $\begin{array}{l}\text { The Hebrew University, Israel } \\
\text { raz@math.huji.ac.il }\end{array}$ \\
\hline Phil Colella & $\begin{array}{l}\text { Lawrence Berkeley Nat. Lab., USA } \\
\text { pcolella@lbl.gov }\end{array}$ & Randall J. LeVeque & $\begin{array}{l}\text { University of Washington, USA } \\
\text { rj1@ amath.washington.edu }\end{array}$ \\
\hline Peter Constantin & $\begin{array}{l}\text { University of Chicago, USA } \\
\text { const@cs.uchicago.edu }\end{array}$ & Mitchell Luskin & $\begin{array}{l}\text { University of Minnesota, USA } \\
\text { luskin@umn.edu }\end{array}$ \\
\hline Maksymilian Dryja & $\begin{array}{l}\text { Warsaw University, Poland } \\
\text { maksymilian.dryja@acn.waw.pl }\end{array}$ & Yvon Maday & $\begin{array}{l}\text { Université Pierre et Marie Curie, France } \\
\text { maday@ann.jussieu.fr }\end{array}$ \\
\hline M. Gregory Forest & $\begin{array}{l}\text { University of North Carolina, USA } \\
\text { forest@amath.unc.edu }\end{array}$ & James Sethian & $\begin{array}{l}\text { University of California, Berkeley, USA } \\
\text { sethian@ math.berkeley.edu }\end{array}$ \\
\hline Leslie Greengard & $\begin{array}{l}\text { New York University, USA } \\
\text { greengard@ cims.nyu.edu }\end{array}$ & Juan Luis Vázquez & $\begin{array}{l}\text { Universidad Autónoma de Madrid, Spain } \\
\text { juanluis.vazquez@uam.es }\end{array}$ \\
\hline Rupert Klein & $\begin{array}{l}\text { Freie Universität Berlin, Germany } \\
\text { rupert.klein@pik-potsdam.de }\end{array}$ & Alfio Quarteroni & $\begin{array}{l}\text { Ecole Polytech. Féd. Lausanne, Switzerland } \\
\text { alfio.quarteroni@epfl.ch }\end{array}$ \\
\hline \multirow[t]{2}{*}{ Nigel Goldenfeld } & $\begin{array}{l}\text { University of Illinois, USA } \\
\text { nigel@uiuc.edu }\end{array}$ & Eitan Tadmor & $\begin{array}{l}\text { University of Maryland, USA } \\
\text { etadmor@cscamm.umd.edu }\end{array}$ \\
\hline & & Denis Talay & $\begin{array}{l}\text { INRIA, France } \\
\text { denis.talay@inria.fr }\end{array}$ \\
\hline
\end{tabular}

\section{PRODUCTION}

production@msp.org

Silvio Levy, Scientific Editor

See inside back cover or msp.org/camcos for submission instructions.

The subscription price for 2015 is US $\$ 85 /$ year for the electronic version, and $\$ 120 /$ year $(+\$ 15$, if shipping outside the US) for print and electronic. Subscriptions, requests for back issues from the last three years and changes of subscribers address should be sent to MSP.

Communications in Applied Mathematics and Computational Science (ISSN 2157-5452 electronic, 1559-3940 printed) at Mathematical Sciences Publishers, 798 Evans Hall \#3840, c/o University of California, Berkeley, CA 94720-3840, is published continuously online. Periodical rate postage paid at Berkeley, CA 94704, and additional mailing offices.

CAMCoS peer review and production are managed by EditFLOW ${ }^{\circledR}$ from MSP.

\section{PUBLISHED BY}

mathematical sciences publishers

nonprofit scientific publishing

http://msp.org/

(C) 2015 Mathematical Sciences Publishers 


\section{Communications in Applied Mathematics and Computational Science}

vol. 10

no. 1

2015

Revisionist integral deferred correction with adaptive step-size control

Andrew J. Christlieb, Colin B. Macdonald, Benjamin W.

ONG and RAYMOND J. SPITERI

An adaptively weighted Galerkin finite element method for boundary value problems

Yifei Sun and Chad R. Westphal

An adaptive finite volume method for the incompressible Navier-Stokes equations in complex geometries

David Trebotich and Daniel T. Graves

High-accuracy embedded boundary grid generation using the divergence theorem

Peter Schwartz, Julie Percelay, Terry J. Ligocki, Hans

Johansen, Daniel T. Graves, Dharshi Devendran, Phillip

Colella and Eli Ateljevich 